\title{
Téoros
}

Revue de recherche en tourisme

\section{Les tables champêtres : une aventure gastronomique différente}

\section{Sylvie Fortin et Marie-France Charette}

Volume 3, numéro 3, novembre 1984

Cuisine québécoise, restauration et tourisme : vers une synergie

féconde

URI : https://id.erudit.org/iderudit/1080775ar

DOI : https://doi.org/10.7202/1080775ar

Aller au sommaire du numéro

Éditeur(s)

Université du Québec à Montréal

ISSN

0712-8657 (imprimé)

1923-2705 (numérique)

Découvrir la revue

Citer cet article

Fortin, S. \& Charette, M.-F. (1984). Les tables champêtres : une aventure gastronomique différente. Téoros, 3(3), 14-19. https://doi.org/10.7202/1080775ar d'utilisation que vous pouvez consulter en ligne.

https://apropos.erudit.org/fr/usagers/politique-dutilisation/ 


\title{
Les tables champêtres: une aventure gastronomique différente
}

\author{
par Sylvie Fortin*
}

Pour les Québécois, un repas "fait à la mai-

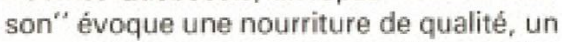
aspect important de la tradition, voire méme un plaisir gastronomique. II n'est donc pas surprenant que la nourriture ait été nommée comme la plus forte source de satisfaction par la clientẻle d'Agricotours ayant fait un séjour à la ferme, et ce dans une proportion de $80 \%$. (Recherche effectuée par trois étudiantes en gestion et intervention touristiques à l'Université du Québec à Montréal en 1982).

En effet, le milieu rural regorge de richesses culinaires traditionnelles, mieux préservees que dans le milieu urbain. De plus, quoi de plus appétissant qu'un mets préparé avec des produits provenant directement du potager et de l'exploitation agricole??

C'est dans cette optique qu'a germé l'idée de pouvoir offrir au public des tables champétres, sur les lieux mêmes de l'exploitation agricole de l'agriculteur et préparées par sa famille. De plus, cette activité nouvelle cadrait parfaitement avec les objectifs de la Fédération des agricotours du Québec qui consistent à promouvoir l'agrotourisme et le tourisme en milieu rural.

Afin de conserver un certain cachet à l'en= treprise, on a pris soin de ne pas donner un caractère commercial au produit.

\section{Historique}

En octobre 1981, le conseil d'administration de la Fédération des agricotours du Québec formait un comité dont le mandat consistait à élaborer une politique opérationnelle d'intervention pour les tables d'hôtes. Cinq personnes composaient ce comité, représentant les organismes suivants: I'Institut de tourisme et d'ho̊tellerie du Québec, le CEGEP de Granby Itechniques de tourismel, l'Office des producteurs de lait du Québec et la Fédération des agricotours du Quếbec.

Ce comité devait faire suite à un projet déjà amorcé par le CODAT (Comité d'orienta-

\footnotetext{
'Sylvie Fortin est agent d'information â la Fédération des agricotours du Qutbec.
}

tion pour le développement de l'agrotourisme) et le MAPAQ (ministère de l'Agriculture, des Pêcheries et de l'Alimentation du Québec). Ce projet s'énonçait comme suit: L'éfablissement d'un réseau de tables d'hotes regionales a titre experimental au Quebec, en conservant le cachet rural, /'accueil typique de l'agrotourisme et /accessibilite pour tous.

On y définissait aussi la table d'hôte comme un repas à prix fixe, servi dans une maison de ferme, suite à une réservation. On y dégustait de la "bonne bouffe" régionale. Un élément essentiel: le service de mets provenant de la ferme de l'hóte pour quelques éléments du menu.

\section{Particularités}

II est clair que la table champêtre (terme qu'on utilise aujourd'hui pour nommer la table d'hôte en milieu rural et membre d'Agricotours) n'est pas un restaurant. Tout d'abord, ce service n'est pas opérationnel de façon quotidienne. II est nécessaire de réserver au moins 48 heures à l'avance. De plus, un minimum de quatre personnes est exigé et l'on ne reçoit qu'un groupe a la fois par repas. Ensuite, le service à la clientẻle est personnalisé: prendre un repas à une table champêtre c'est vivre un échange avec l'hôte et sa famille. II n'est pas question ici de la relation conventionnelle "serveur-client".

L'ambiance dans laquelle se déroule le repas est plus chaude et plus accueillante parce qu'elle revêt un caractère intime du fait mème de se trouver dans le foyer de la famille-höte et de déguster un repas cuisiné par elle. L'aspect "cuisine familiale" a ici son importance meme s'il s'agit d'un repas gastronomique.

\section{Clientèle}

Présentement, selon une étude réalisée par Monique Raymond pour Agricotours en 1982, la clientèle des tables champêtres est composée majoritairement d'adultes ayant un revenu familial annuel d'environ $\$ 30,000$. et provenant de milieux professionnels. On peut expliquer cet état de fait comme suit:
- ces personnes sont déjà sensibilisées aux milieux ruraux;

- les personnes qui désirent tenter cette expérience possedent une auto pour se déplacer et doivent payer le prix du repas (établi à $\$ 15,00$ pour les adultes et à $\$ 8.00$ pour les enfants) et du déplacement.

Cependant, il faut bien avouer que ces explications ne justifient pas I'obligation d'avoir un revenu si élevé pour participer à une table champêtre!

La clientèle provient aussi des grands centres et plus particuliẻrement de la région montréalaise. Des efforts de développement devront être poursuivis afin de sensibiliser les personnes vivant dans les autres régions de la province et plus particulièrement de la région de Québec.

\section{Plan de promotion}

A I'heure actuelle, la promotion la plus efficace des tables champetres réside dans le "bouche à oreille". Cependant, une stratégie de promotion plus systématique devra être mise en oeuvre.

II est clair que la clientèle-cible des tables champêtres ne peut pas être identifiée que par son revenu annuel élevé et ses occupations qualifiées de "professionnelles". L'expérience d'un repas ả une table champềtre paraít intéressante surtout à des gens ouverts, qui aiment vivre des expériences nouvelles. La gastronomie et la recherche de l'alternatif peuvent figurer au nombre des plaisirs qu'aiment s'offrir un grand nombre de personnes dont le revenu annuel est bien inférieur à $\$ 30,000$. Et, tout au contraire, les personnes a haut revenu $n$ 'ont pas nécessairement un faible pour les aventures culinaires non conventionnelles!

Ainsi donc, si une stratégie de promotion doit être mise en place, la clientẻle-cible devra être cernée selon des paramètres failsant référence au degré d'ouverture d'esprit et à l'attirance pour des activités plus ou moins alternatives.

(Suite a la page 19) 


\section{Les tables champêtres:} une aventure gastronomique différente

Enfin, il sera important, dans une perspective publicitaire, de souligner l'aspect peu commun de la nourriture que l'on peut retrouver aux tables champetres. En effet. un certain nombre d'entre elles offrent au consommateurs des produits de culture ou d'élevage peu répandus dans l'industrie de la restauration traditionnelle ou peu accessibles lasperges, produits d'élevage de la chevre, faisan, lapin, etc. I

\section{Un programme en pleine expansion}

On compte à l'heure actuelle 10 tables champêtres réparties dans 8 régions du Québec. Toutes ont été sélectionnées avec soin par la Fédération des agricotours du Québec et approuvées par une personneressource de l'Institut de tourisme et d'hôtellerie du Québec. Une brochure intitulée "La Carte des Cartes" contient tous les renseignements concernant ce programme: les menus détailles de chaque table champêtre avec les coordonnées de l'hóte, les tarifs, les réservations, etc. Elle sera disponible sous peu.

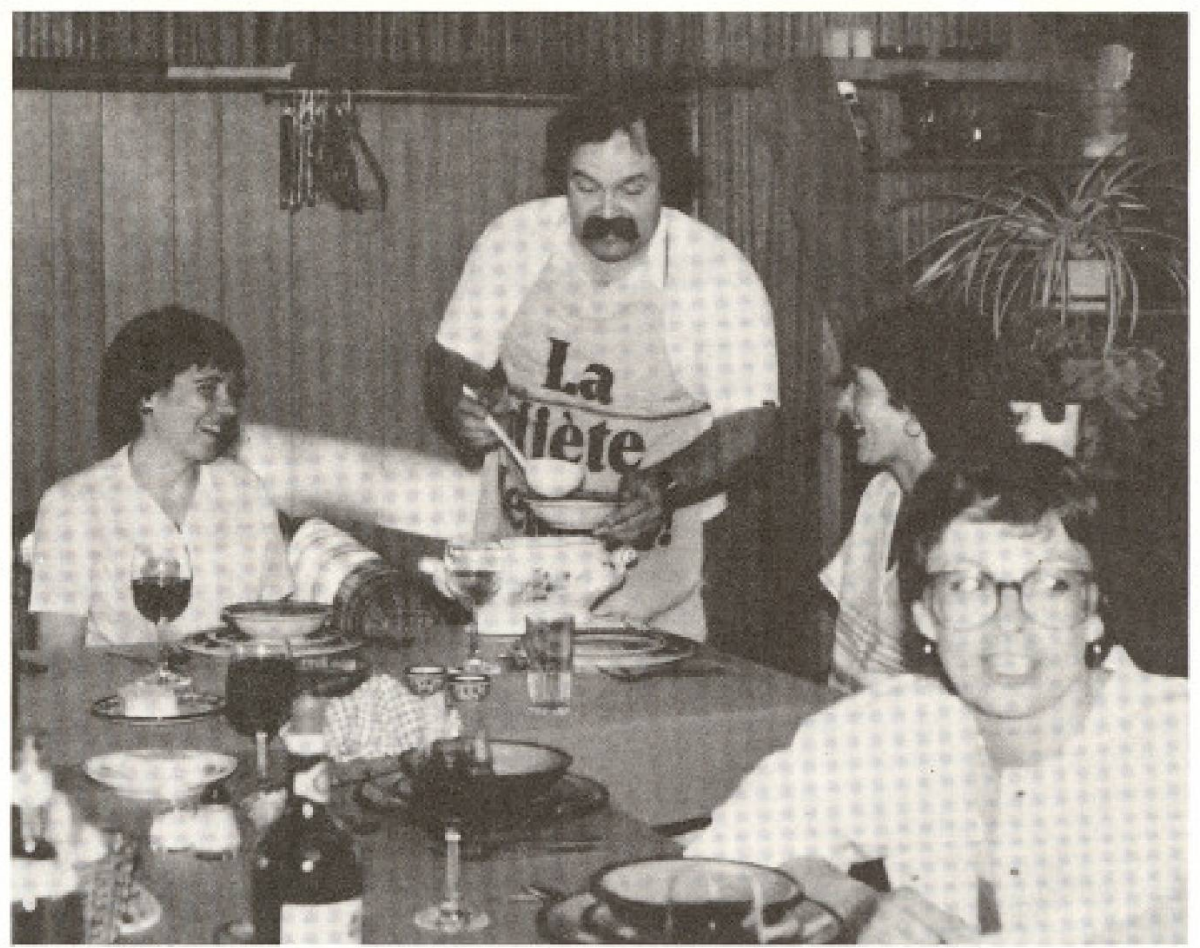

Le service a la clientèle est personnalisé: prendre un repas a une table champêtre, çest vivre un echange avec l'hote et sa famille.

Les tables champêtres, c'est un type de restauration alternatif qu'il faut découvrir. Cette expérience peut ausși s'inscrire à l'intérieur d'un périple qu'on effectue en milieu rural! De plus, votre hôte sera sûrement ravi de vous indiquer quelques beaux coins â visiter dans sa région.

\section{Pourquoi parcourir $100 \mathrm{~km}$ pour aller manger?}

Décrire ce qu'est une table champêtre sans relater l'expérience qu'on y a vécue s'avère impossible. On ne va pas á une table champểtre comme on va au restaurant. II faut tout d'abord aorganisers ce genre de sortie. Les réservations doivent se faire 48 heures â l'avance, soit directement chez l'höte, ou par le biais de Vacances-Familles, I'organisme qui s'occupe des réservations pour tous les programmes d'Agricotours. De plus, la table champêtre exige la présence d'un groupe de quatre ả douze personnes. Pas question d"y aller toute seule pour les besoins d'un article.

Aprẻs m'être pliée ả ces règles, ill me fallut choisir l'endroit et l'hóte. On retrouve des tables champêtres dans huit (8) régions du Québec, un choix s'impose. Personnellement, j'optai pour une région touristiquement attirante, facile d'accès et ả une distance raisonnable de mon point de départ. Chose importante dans la mise en marché de cette activité, la clientéle Idont je suis la représentantel doit défrayer le coût du repas et du déplacement.

Pourquoi parcourir $100 \mathrm{~km}$ pour aller souper? Cela demande une motivation autre que celle de bien manger. Lorsqu'on désire simplement bien manger, on peut généra- lement le faire tout pres de chez soi. Si on décide de parcourir un si long trajet, c'est que quelque chose de spécial nous $y$ motive. Au début, je croyais que le menu à lui seul pouvait justifier cet intérêt. Selon les règlements de la F.A.Q., le menu a prix fixe demeure toujours le meme pendant toute la saison estivale. Le repas doit se composer de produits de la ferme ou tout au moins de la production locale ou régionále. La façon d'apprëter les plats s'avere importante. On utilise les herbes frailches, les fruits et les légumes de saison et on tire profit des secrets culinaires de la maison.

Le réseau comporte des tables champêtres offrant de l'agneau, du faisan, de la chè. vre, du lapin et mëme de la cuisine macrobiotique. De quoi satisfaire tous les goüts. Certains hôtes ont créé leurs propres recettes, chefs-d'oeuvre de la gastronomie rurale. Dans la région Richelieu Rive Sud, vous pouvez goüter quatorze (14) fromages de chèvre, tous fabriqués sur place et offerts en dégustation.

Si le menu est très attírant, ce n'est vraimentqu'à mon arrivée que j'ai compris l'autre raison qui justifie un tel dêplacement: la qualité de l'accueil. Dès l'instant oủ je suis sortie de la voiture, j'ai fait la connais. sance de mes hote et hôtesse. On me fait visiter le jardin et on me présente les animaux domestiques, j'apprends à m'en éloigner ou à leur faire confiance et je-fais tout de suite partie de la famille. On réserve le mème accueil aux amis qui m'accompagnent.

On passe au salon où la table est dressée. et le repas commence. Nous sommes emerveillés à chaque service. Tout est excellent et même... un peu plus encore. Le décor familial est accueillant. $u$ C'est comme si on avait dressé la grande table dans le salon pour recevoir de la visitex. On découvre la composition des mets et on en redemande. Quant aux besoins alcoolisées, la formule «Apportez votre vinw, empruntée à la restauration urbaine est de rigueur. II arrive cependant que I'on vous offre un apéritif maison. Pendant le repas, les hớtes s'affairent au service avec une aisance et une assurance professionnelles.

Bref, il manque peut-ễtre encore des éléments pour que les tables champêtres deviennent une activité importante au sein de l'industrie touristique... mais quand l'hospitalité est au menu, on ne peut demander un meileur départ.

Marie-France Charette 